\title{
Avaliação da disfunção e da incapacidade nas pneumoconioses*
}

\author{
Evaluation of respiratory dysfunction and disability
}

\author{
JOSÉ ALBERTO NEDER ${ }^{1}$, ERICSON BAGATIN², LUIZ EDUARDO NERY³
}

\section{RESUMO}

A determinaçâo das conseqüências funcionais (disfunção) e do seu impacto na vida diária (incapacidade) são aspectos centrais da avalição de pacientes com doença ocupacional respiratória. A presente revisão apresenta as bases fundamentais para o entendimento dos instrumentos utilizados para a definição do grau de disfunção, incluindo aspectos clínicos, funcionais pulmonares e, em algumas circunstâncias, da capacidade de exercício. Em especial, apresenta-se uma classificação multifatorial do grau de disfunção com o intuito de nortear a concessão ou não de benefícios previdenciários em nosso meio.

Descritores: Avaliaçao da deficiência; Testes de função respiratórias; Doenças respiratórias; Doenças ocupacionais; Exposição ocupacional; Condições de trabalho

\begin{abstract}
The determination of functional consequences (dysfunction) and their impact on daily life (incapacitation) is central to the evaluation of patients with occupational respiratory diseases. The present review addresses the fundamentals underlying the instruments used to determine the degree of dysfunction, including clinical aspects, as well as those related to pulmonary function and, in some circumstances, exercise tolerance. In particular, a multifactorial system of classifying the degree of dysfunction is presented, with the objective of informing decisions related to the awarding of retirement benefits in Brazil.
\end{abstract}

Keywords: Disability Evaluation; Respiratory function tests; Respiratory tract diseases; Occupational diseases; Occupational exposure; Working conditions

\section{INTRODUÇÃO}

Os têrmos impairment e disability, secundários a doença respiratória, utilizados nas publicações da American Medical Association ${ }^{(1-3)}$ e da American Thoracic Society, ${ }^{(4-5)}$ traduzidos como disfunção e incapacidade, referem-se a diferentes conceitos que devem ser claramente entendidos. Assim, disfunção respiratória é definida como uma condição puramente médica, resultante de uma anormalidade funcional. Ela pode ser estável ou não, temporária ou permanente. A disfunção pode ter graduação variável e é habitualmente avaliada por testes de função pulmonar (mas não exclusivamente).

Em contraste, a American Thoracic Society define incapacidade como o efeito global da disfunção na vida do paciente. Assim, a incapacidade não é relacionada apenas a condições médicas, mas envolve fatores mais complexos, tais como: idade, sexo, dimensões corpóreas, educação, condições psicológica e socioeconômica e requerimen-

* Trabalho realizado na da Disciplina de Pneumologia da Escola Paulista de Medicina da Universidade Federal de São Paulo - UNIFESP - São Paulo (SP) Brasil e na Faculdade de Ciências Médicas da Universidade Estadual de Campinas UNICAMP - Campinas (SP) Brasil.

1. Professor Adjunto Livre Docente da Disciplina de Pneumologia da Escola Paulista de Medicina (EPM) da Universidade Federal de São Paulo - UNIFESP - São Paulo (SP) Brasil.

2. Professor Assistente Doutor da Área de Saúde Ocupacional do DMPS da Faculdade de Ciências Médicas da Universidade Estadual de Campinas - UNICAMP - Campinas (SP) Brasil.

3. Professor Adjunto Doutor e Chefe da Disciplina de Pneumologia do Departamento de Medicina da Escola Paulista de Medicina (EPM) da Universidade Federal de São Paulo - UNIFESP - São Paulo (SP) Brasil.

Endereço para correspondência: José Alberto Neder. Rua Professor Francisco de Castro, 54 - CEP 04050-020, São Paulo, SP, Brasil. E-mail: sefice@pneumo.epm.br 
tos energéticos da ocupação. Outro conceito de cunho médico-administrativo é o grau de empregabilidade (employability) de determinado indivíduo. Não raramente, o médico é chamado a opinar se um indivíduo pode ou não ser empregado, ou manter-se em determinada função. Sua opinião deve considerar a capacidade do examinado em ir e vir do trabalho, permanecer no local pelo tempo necessário e realizar com segurança, para si e para os colegas, as tarefas propostas pelo empregador. Assim, se um indivíduo com uma anormalidade médica tem ainda a capacidade de suportar confortavelmente as exigências do deslocamento e da função, ele pode ainda ser considerado empregável. Neste contexto, as informações médicas serão utilizadas como esteio do raciocínio legal, mas serão cotejadas com diversos outros aspectos socioergonômicos.

\section{AVALIAÇÃO DA DISFUNÇÃO}

$\mathrm{Na}$ presença de entidade nosológica ocupacional com substrato anatomofuncional estável ou potencialmente estável - silicose, asbestose, pneumoconiose do trabalhador do carvão - podem ser empregados no diagnóstico e quantificação do dano respiratório: avaliação clínica, exame radiográfico torácico, tomografia computadorizada, testes de função pulmonar e testes cardiopulmonares de exercício. ${ }^{(6-7)}$

\section{Avaliação clínica}

A obtenção de uma história clínica detalhada e completa deve sempre preceder a investigação laboratorial. Como já salientado, deve-se enfatizar a história ocupacional, ambiental, mórbida pregressa e atual, dos hábitos de lazer e tabágica.

Os principais sintomas respiratórios - dispnéia, tosse, expectoração, sibilância - devem ser cuidadosamente inquiridos, observando-se a relação temporal com a história de exposições ocupacionais ou não. ldealmente, todos os sintomas deveriam ser objetivamente graduados por esquema classificatório específico. Para a dispnéia, tal conduta é essencial (Tabela 1).

0 exame físico deve ser geral, com avaliação pormenorizada dos sinais associados à possível doença respiratória subjacente. Especificamente, deve-se atentar para o padrão respiratório e posturas corporais peculiares, utilização da muscula- tória ventilatória acessória e respiração com lábios semicerrados. Todos os ruídos adventícios deverão ser descritos, localizados topograficamente, quantificados e situados quanto à fase do ciclo respiratório. De fundamental importância, ainda, é a procura de sinais não torácicos ou sistêmicos que indiquem patologia pulmonar avançada, os quais modificarão substancialmente a graduação da disfunção: cianose ou pletora, baqueteamento digital, dilatação venosa jugular, edema de membros inferiores e asteríxis.

\section{Radiograma}

No estabelecimento diagnóstico e prognóstico de pneumopatias ocupacionais por poeiras minerais é crucial a leitura do radiograma de tórax seguindo os radiogramas padrão da Classificação Internacional de Radiografias das Pneumoconioses. ${ }^{(8)}$ Embora apenas nos casos com maior acometimento radiográfico exista uma discreta correlação entre os achados clínicos, radiológicos e funcionais respiratórios, a uniformização da linguagem interobservador é fundamental para a quantificação do dano anatômico e o seguimento longitudinal.

A avaliação radiológica de outros aspectos como hiperinsuflação pulmonar, diminuição da vascularização, presença de bolhas, achatamento diafragmático, espessamento pleural, anormalidades cardíacas direitas, alterações esqueléticas torácicas (principalmente cifoescoliose avançada) podem sugerir comprometimento respiratório significativo.

\section{Tomografia computadorizada de alta resolução do tórax}

Nos últimos anos, tem sido utilizada com maior frequência a tomografia computadorizada e, principalmente, a tomografia computadorizada de alta resolução no diagnóstico das alterações pleuropulmonares associadas ao asbesto. Embora a baixa disponibilidade e o custo elevado ainda limitem o uso da tomografia computadorizada de alta resolução e cortes finos no diagnóstico, trata-se de um exame praticamente indispensável na detecção das imagens intersticiais iniciais, no diagnóstico das anormalidades pleurais e na investigação de prováveis neoplasias de pleura e pulmão. Neste contexto, a tomografia helicoidal apresenta clara vantagem em relação às técnicas tradicionais. Ela deve ser obrigatoriamente realizada em decúbito ventral, sendo identificados na asbestose: espessamento 
TABELA 1

Classificação de disfunção no repouso e no exercício para doenças respiratórias com anormalidade funcional estável

\begin{tabular}{|c|c|c|c|c|}
\hline$\overline{\text { Grau }}$ & $\mathrm{I}$ & II & III & IV \\
\hline Variável & Sem disfunção & Disfunção leve & Disfunção moderada & Disfunção acentuada \\
\hline a) Sintoma & & Andando rápido & Andando no plano & Andando devagar \\
\hline Dispnéia & Ausente & $\begin{array}{l}\text { no plano ou subindo } \\
\text { ladeira devagar }\end{array}$ & $\begin{array}{l}\text { com pessoa da mesma } \\
\text { idade ou subindo o lance } \\
\text { de escada }\end{array}$ & $\begin{array}{l}\text { no plano } 100 \text { metros } \\
\text { esforços menores ou } \\
\text { mesmo no repouso }\end{array}$ \\
\hline \multicolumn{5}{|c|}{ b) Mecânica ventilatória } \\
\hline${ }^{*} \% \mathrm{CVF}^{* * *}$ & $>\mathrm{Ll} \mathrm{nl} \mathrm{\#}$ & $60-\mathrm{Ll} \mathrm{nl}$ & $51-59$ & $\leq 50$ \\
\hline$\% \mathrm{VEF}_{1} * * *$ & $>\mathrm{Ll} \mathrm{nl} \mathrm{\#}$ & $60-\mathrm{Ll} \mathrm{nl}$ & $41-59$ & $\leq 40$ \\
\hline $\mathrm{VEF}_{1} / \mathrm{CVF}(\%)$ & $>\mathrm{Ll} \mathrm{nl} \mathrm{\#}$ & $60-\mathrm{Ll} \mathrm{nl}$ & $41-59$ & $\leq 40$ \\
\hline$\% \mathrm{CPT}^{* *}$ & $\geq 80$ & $70-79$ & $61-69$ & $\leq 60$ \\
\hline \multicolumn{5}{|c|}{ c) Troca gasosa } \\
\hline${ }^{*} \% \mathrm{D}_{\mathrm{L}} \mathrm{CO}$ & $\geq 70$ & $60-69$ & $41-59$ & $\leq 40$ \\
\hline $\begin{array}{l}\mathrm{PaO}_{2}(\mathrm{mmHg}) \\
\text { d) Exercício }\end{array}$ & $>\mathrm{Ll} \mathrm{nl} \mathrm{\#}$ & $60-\mathrm{Ll} n \mathrm{l}$ & $55-59$ & $<55$ \\
\hline $\begin{array}{l}\text { *VO }{ }_{2} \text { máx } \\
\text { (\% previsto) }\end{array}$ & $\geq 80 \#$ & $60-79$ & $41-59$ & $\leq 40$ ou $<1 \mathrm{~L} / \mathrm{min}$ \\
\hline $\begin{array}{l}\mathrm{VO}_{2} \operatorname{máx} \\
(\mathrm{mL} / \mathrm{min} / \mathrm{kg} \text { ou }\end{array}$ & $\underset{/ \min )}{\geq 25}$ & $20-24$ & $16-19$ & $\leq 15$ ou $<1 \mathrm{l} / \mathrm{min}$ \\
\hline
\end{tabular}

Modificada da American Medical Association, 1984, 1988, 1993; American Thoracic Society, 1982, 1986; Nery, Neder, Bagatin, 1994. * Os valores previstos da normalidade seguem as normas da Sociedade Brasileira de Pneumologia e Tisiologia.

** São as variáveis fundamentais para a graduação do distúrbio restritivo(ver o texto); *** É a variável fundamental para a graduação do distúrbio obstrutivo; \# Ll nl: Limite inferior da normalidade - limite inferior do intervalo de confiança de 95\% (X - 1,64 x desviopadrão); NOTA: Graduar de acordo com o teste mais alterado; CVF: capacidade vital forçada; $\mathrm{VEF}_{1}$ : volume expiratório forçado no primeiro segundo; CPT: capacidade pulmonar total; DLCO: capacidade de difusão pulmonar para o monóxido de carbono; $\mathrm{SaO}_{2}$ : saturação arterial de oxihemoglobina; $\mathrm{PaO}_{2}$ : pressão parcial de oxigênio no sangue arterial; $\mathrm{VO}_{2}$ máx: consumo máximo de oxigênio.

dos septos interlobulares, bandas que se estendem da pleura ao parênquima, pontos e linhas subpleurais e, nos estágios mais avançados, anormalidades císticas tipo faveolamento e bronquiolectasias de tração (sinais de alta predição de fibrose intersticial). Estas alterações são habitualmente localizadas nas regiões periféricas e posteriores dos lobos inferiores. Vários trabalhos, inclusive em nosso meio, têm constatado a maior sensibilidade da tomografia computadorizada de alta resolução em relação ao radiograma de tórax na identificação das alterações intersticiais. Por outro lado, num número expressivo de indivíduos com alterações radiológicas (1/0 ou mais), a tomografia computadorizada de alta resolução não mostrou comprometimento intersticial pulmonar, o que confirma a maior especificidade deste exame. ${ }^{(9)}$

\section{Testes funcionais respiratórios}

A espirometria constitui o teste de maior importância prática para o estudo do desempenho venti- latório toracopulmonar. Destaca-se a importância de variáveis simples e reprodutiveis, obtidas a partir da manobra expiratória forçada: capacidade vital forçada, volume expiratório forçado no primeiro segundo e a relação entre eles. No propósito de graduar a disfunção, não se recomenda a utilização de outros parâmetros espirométricos de maior variabilidade inter e intrapessoal e padronização ainda deficiente, como os fluxos meso e tele-expiratórios. Os critérios de aceitabilidade e reprodutibilidade das curvas, análise dos resultados, valores de referência e demais aspectos técnicos devem seguir rigorosamente as recomendações das Diretrizes para Testes de Função Pulmonar da Sociedade Brasileira de Pneumologia e Tisiologia. ${ }^{(10)}$ Deve-se atentar para o fato de que o diagnóstico de distúrbio restritivo baseia-se na redução da capacidade pulmonar total (CPT). A redução isolada da capacidade vital forçada (CVF) não é patognomônica de distúrbio restritivo, já que diversas condições podem reduzi-la, inclusive a obstrução das 
vias aéreas. $\mathrm{Na}$ indisponibilidade da medida da CPT (preferencialmente por pletismografia corporal), a CVF pode ser utilizada, com reservas, para definir distúrbio restritivo.

A capacidade de difusão pulmonar para o monóxido de carbono traz informações sensíveis a respeito da integridade anatomofuncional da troca gasosa através da membrana alveolocapilar. Em nosso meio, embora poucos serviços disponham deste teste, é de importância fundamental a avaliação da capacidade de difusão pulmonar para o monóxido de carbono na identificação e gradação da disfunção e incapacidade das pneumoconioses. Caso sua mensuração seja disponível, a técnica de respiração única deverá ser preferida, e ser realizada com rígidos padrões técnicos e após no mínimo oito horas sem o uso do tabaco. ${ }^{(11)}$

As determinações das pressões parciais de oxigênio e de dióxido de carbono no sangue arterial periférico apresentam considerável variabilidade interindividual. Elas podem estar ainda preservadas na vigência de comprometimento anatomofuncional, apresentando menor correlação com a tolerância ao exercício do que o volume expiratório forçado no primeiro segundo. Embora menos sensíveis, alterações significativas da pressão parcial de oxigênio no sangue arterial ou da pressão parcial de dióxido de carbono no sangue arterial são específicas de comprometimento ventilatório ou das trocas gasosas em pacientes com disfunção fixa por pneumoconioses. A medida não invasiva da saturação de oxihemoglobina deve ser encarada apenas como uma estimativa da oxigenação sanguínea, devendo seus resultados serem confirmados com a aferição invasiva da pressão parcial de oxigênio no sangue arterial.

\section{Teste de exercício cardiopulmonar}

0 teste de exercício cardiopulmonar, com a determinação direta das respostas ventilatórias, cardiovasculares e metabólicas, é um procedimento sensível para avaliacão e quantificação objetiva da limitação funcional. Uma redução da tolerância ao exercício aeróbio é definida quando o consumo de oxigênio no pico de uma atividade limitada por sintomas ( $\mathrm{VO}_{2}$ max) encontra-se abaixo do limite inferior da normalidade para indivíduos de mesma etnia, sexo, idade, altura, massa muscular e nível de atividade física regular.

0 teste de exercício cardiopulmonar é indicado para os casos nos quais a queixa de dispnéia é desproporcional à disfunção pulmonar aferida no repouso, e nos indivíduos que exercem atividades com esforço sustentado de grau moderado ou com picos de esforço intenso. Pode ainda ser utilizado para avaliar a tolerância ao esforço do indivíduo no desempenho do seu trabalho, numa análise comparativa com o requerimento energético de várias atividades recreacionais, domésticas e ocupacionais. ${ }^{(12)} \mathrm{A}$ gasometria arterial no exercício, mesmo em indivíduos com testes de repouso normais, pode revelar, precocemente, alterações nas trocas gasosas.

Para uma correta interpretação do teste de exercício cardiopulmonar, devemos considerar os seguintes aspectos: certificar-se da presença de relação causal entre a disfunção aeróbia e a doença respiratória em questão; definir-se o grau de perda funcional aeróbia, numa análise comparativa com os valores previstos de $\mathrm{VO}_{2}$ max para indivíduos de mesmo sexo, idade, dimensão corpórea e nível de atividade física habitual (porcentagem do $\mathrm{VO}_{2}$ max previsto); determinar-se o grau de capacidade aeróbia remanescente, num enfoque comparativo com as prováveis demandas ocupacionais ou da vida diária ( $\mathrm{VO}_{2}$ máx, $\mathrm{L} / \mathrm{min}$ ou $\mathrm{mL} / \mathrm{min} / \mathrm{kg}$ ); nos indivíduos idosos e com peso acima do esperado para a altura, a expressão em $\mathrm{mL} / \mathrm{min} / \mathrm{kg}$ deve ser evitada, já que tende a subestimar grosseiramente a capacidade atual de exercício. ${ }^{(13)}$

\section{Outros exames}

De acordo com situações clínicas específicas, outros exames opcionais poderão ser empregados, tanto no contexto diagnóstico, quanto da avaliação da disfunção: eletrocardiograma, ecocardiograma, estudo hemodinâmico, exames hematológicos e bioquímicos, exame polissonográfico e/ou monitorização noturna da oximetria, entre outros. Estes exames poderão ser úteis para a comprovação de distúrbios sugeridos pela avaliação clínica e documentação objetiva de achados que possam influenciar na graduação da disfunção.

\section{CLASSIFICAÇÕES DE DISFUNÇÃO}

As tentativas de graduar a disfunção respiratória em classes de progressiva gravidade remontam ao início do século passado. Entretanto, apenas em 1982 a American Thoracic Society propôs um esquema classificatório de ampla aceitação desenvol- 
vido para caracterizar disfunção grave, baseado na espirometria, na capacidade de difusão pulmonar ao monóxido de carbono e na avaliação da capacidade de exercício. ${ }^{(4)}$ Assim, a disfunção foi definida espirometricamente como grave quando a capacidade vital forçada fosse inferior a 50\% do previsto e o volume expiratório forçado no primeiro segundo e a relação entre ele e a capacidade vital forçada estivessem abaixo de $40 \%$ do previsto. Adicionalmente, valores de capacidade de difusão pulmonar para o monóxido de carbono menores do que $40 \%$ do previsto eram indicativos de disfunção respiratória grave. Nos casos em que a espirometria e/ou a capacidade de difusão pulmonar para o monóxido de carbono estavam alterados, mas sem disfunção grave (ou seja, indivíduos com distúrbio ventilatório leve a moderado) era indicado o teste de exercício. $\mathrm{Na}$ avaliação cardiorrespiratória no exercício aeróbio, foram considerados como sugestivos de disfunção grave os valores de $\mathrm{VO}_{2}$ máx abaixo de $15 \mathrm{ml} / \mathrm{min} / \mathrm{kg}$, ou caso $30 \%$ a $40 \%$ do $\mathrm{VO}_{2} \max$ obtido fosse insuficiente para o indivíduo suprir as demandas enegético-metabólicas de uma jornada diária de oito horas.

Em 1984, 1988 e 1993 a American Medical Association $^{(1-3)}$ e em 1986 a American Thoracic Society ${ }^{(5)}$ introduziram o conceito de graus intermediários de disfuncão causada por doenças respiratórias. As classificações são similares e utilizam variáveis espirométricas, o $\mathrm{VO}_{2}$ máx e a capacidade de difusão pulmonar para o monóxido de carbono para definir graus leve, moderado e acentuado de comprometimento. A classificação da American Medical Association, além de introduzir a graduação da dispnéia, considera, na análise espirométrica, o limite inferior da normalidade como correspondente ao limite inferior do intervalo de confiança de 95\% do valor previsto.

Baseando-se nestas referências clássicas, e na experiência com a avaliação funcional no repouso e exercício de pacientes com pneumoconiose, propomos uma classificação de disfunção respiratória que incorpora os avanços obtidos nessa área. Desta forma, como explicitado na Tabela 1, os achados clínicos, funcionais pulmonares de repouso e, ocasionalmente, de exercício, servirão de base para a graduação da perda funcional.

$\mathrm{Na}$ análise do $\mathrm{VO}_{2}$ máx, quando o objetivo for orientar a concessão de benefício previdenciário, a perda funcional $\left(\mathrm{VO}_{2}\right.$ máx, porcentagem do pre- visto) deve ser preferida em relação à capacidade remanescente $\left(\mathrm{VO}_{2}\right.$ máx em valores absolutos ou corrigidos para o peso total nos eutróficos ou peso magro nos indivíduos em sobrepeso). Em situações de admissão, afastamento e recolocacão ocupacional, ambas devem ser analisadas.

A presença das seguintes condições indica sempre disfunção acentuada, independentemente da graduação sugerida pela classificação: cor pulmonale; síndrome neoplásica de qualquer origem; hipoxemia arterial no repouso - pressão parcial de oxigênio no sangue arterial $<60 \mathrm{mmHg}$ ou saturação arterial de oxihemoglobina $<90 \%$, se associadas ao cor pulmonale, hipertensão pulmonar ou poliglobulia, e pressão parcial de oxigênio no sangue arterial de repouso ou exercício $<55 \mathrm{mmHg}$ ou saturação arterial de oxihemoglobina $<88 \%$, independentemente de outros achados; obstrução permanente de vias aéreas superiores, associada à hipercapnia.

A presença de distúrbio respiratório do sono deve ser cuidadosamente avaliada, e requer estudo polissonográfico para a precisa quantificação da disfunção. Deve-se observer se a terapia clínica e/ou cirúrgica foi aplicada em toda sua extensão, antes de definir dano funcional permanente. Deve-se atentar também para condições ocupacionais especiais, que podem elevar o grau de disfunção, como o uso de protetores respiratórios ou trabalho em ambiente poluído, de um paciente com disfunção respiratória já diagnosticada.

Admitindo-se que, mesmo nas condições ditas fixas, a disfunção no repouso e exercício pode modificar-se longitudinalmente (com ou sem intervenção terapêutica), aconselha-se a repetição das medidas avaliadoras em intervalo variável de meses a anos, dependendo da doença de base e quadros intercorrentes.

\section{REFERÊNCIAS}

1. American Medical Association. The respiratory system. In: American Medical Association. Guides to the evaluation of permanent impairment. 2nd ed. Chicago: AMA: 1984. p. 85-101.

2. American Medical Association. The respiratory system. In: American Medical Association. Guides to the evaluation of permanent impairment. 3rd ed. Chicago: AMA; 1988. p. 107-18.

3. American Medical Association. The respiratory system. In: American Medical Association. Guides to the evaluation of permanent impairment. 4th ed. Chicago: American Medical Association; 1993. p. 115-29. 
4. American Thoracic Society, medical section of the American lung Association. Evaluation of impairment/ disability secondary to respiratory disease. Am Rev Respir Dis. 1982;126(5):945-51.

5. American Thoracic Society. Evaluation of impairment/ disability secondary to respiratory disorders. Am Rev Respir Dis. 1986;133(6):1205-9.

6. Nery LE, Neder JA, Bagatin E. Avaliação da disfunção e da incapacidade secundária a pneumopatias ocupacionais. J Pneumol. 1994;20(4 n. esp):182-92.

7. Neder JA, Nery LE, Bagatin E. Avaliação da disfunção, incapacidade e dos aspectos legais da doença ocupacional respiratória. In: Cukier A, Nakatani J, Morrone N, editors. Atualização e reciclagem. 2a ed. São Paulo: Atheneu; 1998. p.57-67.

8. International Labour Office. Guidelines for the use of ILO international classification of radiographs of pneumoconiosis. Geneva: Occupational Safety and Health Sciences; 1980.
(Occupational Safety of Health Services, 22).

9. Sette A, Neder JA, Nery LE, Kavakama J, Rodrigues RT, Terra-Filho M, et al. Thin-section CT abnormalities and pulmonary gas exchange impairment in workers exposed to asbestos. Radiology. 2004;232(1):66-74.

10. Sociedade Brasileira de Pneumologia e Tisiologia. Diretrizes para testes de função pulmonar. J Pneumol. 2002;28(supl 3):S1-82.

11. Neder JA, Andreoni S, Peres C, Nery LE. Reference values for lung function tests. Ill. Carbon monoxide diffusing capacity (transfer factor). Braz J Med Biol Res. 1999;32(6):729-37.

12. Neder JA, Nery LE. Fisiologia do exercício: teoria e prática. São Paulo: Artes Médicas; 2003

13. Neder JA, Nery LE, Bagatin E, Lucas SR, Ancao MS, Sue DY. Differences between remaining ability and loss of capacity in maximum aerobic impairment. Braz J Med Biol Res. 1998;31(5):639-46. 